\title{
Temperature-Controlled Chameleonlike Cloak
}

\author{
Ruiguang Peng, ${ }^{1}$ Zongqi Xiao, ${ }^{1}$ Qian Zhao, ${ }^{1, *}$ Fuli Zhang, ${ }^{2}$ Yonggang Meng, ${ }^{1}$ Bo Li, ${ }^{3}$ Ji Zhou, ${ }^{3}$ \\ Yuancheng Fan, ${ }^{2}$ Peng Zhang, ${ }^{4}$ Nian-Hai Shen, ${ }^{4}$ Thomas Koschny, ${ }^{4}$ and Costas M. Soukoulis ${ }^{4,5}$ \\ ${ }^{1}$ State Key Laboratory of Tribology, Department of Mechanical Engineering, \\ Tsinghua University, Beijing 100084, China \\ ${ }^{2}$ Key Laboratory of Space Applied Physics and Chemistry, \\ Ministry of Education and Department of Applied Physics, School of Science, \\ Northwestern Polytechnical University, Xi'an 710072, China \\ ${ }^{3}$ State Key Laboratory of New Ceramics and Fine Processing, \\ School of Materials Science and Engineering, Tsinghua University, Beijing 100084, China \\ ${ }^{4}$ Ames Laboratory and Department of Physics and Astronomy, \\ Iowa State University, Ames, Iowa 50011, USA \\ ${ }^{5}$ Institute of Electronic Structure and Lasers (IESL), FORTH, 71110 Heraklion, Crete, Greece
}

(Received 13 October 2016; published 21 March 2017)

\begin{abstract}
Invisibility cloaking based on transformation optics has brought about unlimited space for reverie. However, the design and fabrication of transformation-optics-based cloaks still remain fairly challenging because of the complicated, even extreme, material prescriptions, including its meticulously engineered anisotropy, inhomogeneity and singularity. And almost all the state-of-the-art cloaking devices work within a narrow and invariable frequency band. Here, we propose a novel mechanism for all-dielectric temperature-controllable cloaks. A prototype device was designed and fabricated with $\mathrm{SrTiO}_{3}$ ferroelectric cuboids as building blocks, and its cloaking effects were successfully demonstrated, including its frequency-agile invisibility by varying temperature. It revealed that the predesignated cloaking device based on our proposed strategy could be directly scaled in dimensions to operate at different frequency regions, without the necessity for further efforts of redesign. Our work opens the door towards the realization of tunable cloaking devices for various practical applications and provides a simple strategy to readily extend the cloaking band from microwave to terahertz regimes without the need for reconfiguration.
\end{abstract}

DOI: 10.1103/PhysRevX.7.011033

Subject Areas: Materials Science, Metamaterials

\section{INTRODUCTION}

An invisible cloak renders a volume effectively invisible to incident radiation [1,2]. The combination of the form invariance of coordinate transformation and artificial metamaterials makes the implementation of such a cloak possible $[3,4]$. Even beyond the realm of electromagnetic waves [5-7], coordinate-transformation-based cloaks for various physical fields have been theoretically predicted and experimentally demonstrated in the past 10 years, such as in direct currents [8], acoustic waves [9], heat flows $[10,11]$, elastic waves [12], matter waves [13], and multiphysics cloaks $[14,15]$. In addition, scattering-cancellationbased cloaks, such as plasmonic cloaks [16] and mantle cloaks [17], have also been developed. Compared to transformation-optics-based cloaks, scattering cancellation

\footnotetext{
* Corresponding author. zhaoqian@mail.tsinghua.edu.cn

Published by the American Physical Society under the terms of the Creative Commons Attribution 4.0 International license. Further distribution of this work must maintain attribution to the author(s) and the published article's title, journal citation, and DOI.
}

has relatively simpler electromagnetic parameters. However, to conceal objects of dimensions comparable to or larger than the wavelength, multilayered structures of high complexity are necessary to compensate the higher-order mode scattering.

An ideal transformation-optics cloak can theoretically make any object perfectly invisible. However, it is still particularly difficult to design and obtain the required constitutive materials that have the anisotropic, inhomogeneous, and sometimes even extreme electromagnetic parameters, thus hindering the development of such a cloak. Nevertheless, by utilizing different transformation functions or specific polarizations [18,19], researchers have explored invisibility cloaks with simplified parameters. For example, the column cloak was first experimentally demonstrated using the unidirectional magnetic response of metallic split ring resonators (SRRs) [5]. In a more complex system, the anisotropic permeability provided by SRRs and corrugated transmission lines has been utilized to realize a unidirectional rhombic cloak [20], in which the strong magnetic coupling in two perpendicular directions was avoided. In addition, it has been known that the conformal or quasiconformal mapping method [21] could reduce the dependence of the cloak on the complicated 
magnetic parameters. A carpet cloak, which was constructed based on this method [22,23], can make an object sitting on the ground invisible. However, requiring a gradient index within the material, which is less than that of the environment in certain regions, it might be difficult for this to work directly in air. Furthermore, the sophisticated electromagnetic parameters obtained around the LC (a circuit consisting of an inductance $\mathrm{L}$ and a capacitance C) resonance frequency of metallic structures are intrinsically dispersive, thus determining the narrow operating band of most reported cloaks. In addition, the mutual coupling between the anisotropic responses of metallic SRRs makes precise material parameter control even more difficult. In other words, any change in material parameters would require the cloak to be entirely redesigned. Alternatively, a frequency-tunable cloak, by means of applying an external field, may offer a possible solution; however, the electromagnetic parameters of the material have to anisotropically change in order to reach the required values for another working frequency.

Recently, several actively controlled cloaks have been reported, such as the active scattering cancellation electromagnetic cloak with a current field [24], tunable plasmonic cloaks with an external magnetic field [25], the switchable transformation thermotics cloak controlled by temperature [26], and the tunable transformation-dc illusion cloak controlled by optical intensity [27]. However, a frequencytunable transformation-optics cloak has not yet been demonstrated because of the challenge of complicated anisotropic electromagnetic parameters. As an Ohmic loss-free alternative to metallic structures, dielectric metamaterials $[28,29]$ based on the Mie resonance of ferroelectric ceramic cuboids have been employed to readily realize the required electromagnetic parameters. More importantly, with the help of external stimuli, the permittivity of the ferroelectric ceramic can be controlled, thereby tuning the Mie resonance of the cuboids, leading to an interesting adjustable wave-control device [30]. Herein, we report a simple design methodology for a unidirectional all-dielectric temperature-tunable transformation-optics cloak and experimentally verify its feasibility.

\section{ALL-DIELECTRIC UNIDIRECTIONAL METAMATERIAL CLOAK}

The theory of transformation optics provides us with the guidelines in designing unidirectional rhombic cloaks with different angles (see Figs. S1-S3 in Ref. [31]). Accordingly, the required electromagnetic parameters for a TE-polarized rhombic cloak with interior angles $\alpha=30^{\circ}$ and $\beta=60^{\circ}$ are $\bar{\mu}_{x}=2.22, \bar{\mu}_{y}=0.45$ (in-plane components of permeability), and $\bar{\epsilon}_{z}=1.5$ (out-of-plane component of permittivity), respectively (Fig. 1). In contrast to the complicated design of metallic units adopted by Landy and Smith in Ref. [20], we propose using pure dielectric Mie particles in cuboid shape to construct the expected
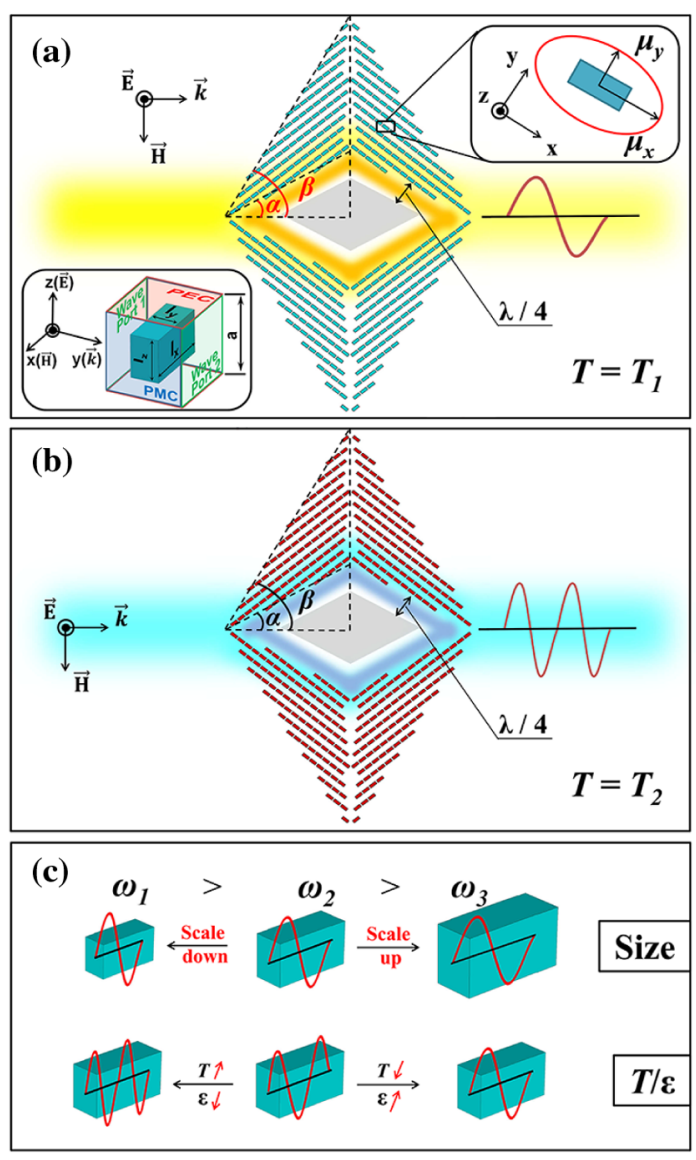

FIG. 1. Schematic of the chameleonlike all-dielectric cloak. The cloak can conceal the object under different temperatures, and the invisible frequency can be adjusted from lower frequency (lower temperature $T_{1}$ ) (a), to higher frequency (higher temperature $T_{2}$ ) (b). The cloak is constructed from dielectric cuboids, which can realize the required anisotropic electromagnetic parameters. A TE wave polarized along the $z$ direction is incident from the left. A quarter-wave separation between the conducting quadrangular and the inner cloak boundaries is designated to simulate a perfect magnetic conductor boundary. The inset shows unit cells used to calculate the effective electromagnetic parameters. (c) Two methods to adjust the working frequency of the cloak. The first is by scaling the size, and the second is by changing the temperature of the dielectric cuboids.

unidirectional cloak, integrating the superiorities in both the simplicity and the flexibility of tunable response. The so-called chameleonlike cloak, which possesses cloaking effects with working frequencies controllable by the external stimuli (temperature), is shown in Fig. 1. In addition to external stimuli, the working frequency can shift higher or lower by scaling down or up the dimensions of the cuboid. However, how can the electromagnetic parameters of the cuboids in different directions still satisfy the required parameters at a new frequency while applying external stimuli or scaling the dimensions? It is a challenge, and we will reveal the mechanism to achieve this below. 


\section{MECHANISM OF CHAMELEONLIKE ALL-DIELECTRIC CLOAK}

The first-order Mie magnetic-dipole-resonance frequency of dielectric cuboids [32] is

$\omega_{0, k}=\pi c\left(\epsilon_{a}\right)^{-1 / 2}\left[\left(1 / l_{i}\right)^{2}+\left(1 / l_{j}\right)^{2}+\left(\delta_{k} / l_{k}\right)^{2}\right]^{1 / 2}$,

where $c$ is the speed of light in free space, $\epsilon_{a}$ is the relative permittivity of Mie particles (intrinsic magnetic response is neglected), $l_{i}$ and $l_{j}$ represent the dimensions of the rectangular cross section $(i-j$ plane) perpendicular to the magnetic vector $\vec{H}, l_{k}$ is the cuboid size along $\vec{H}$, and $\delta_{k}$ $\left(0<\delta_{k}<1\right)$ denotes a fraction of a half-wavelength in the $l_{k}$ direction, subjected to the constraint

$$
\left(\delta_{k} / l_{k}\right)^{2}=\left(\epsilon_{a}-1\right)\left[\omega_{0, k}^{2} /\left(\pi^{2} c^{2}\right)\right] \cos ^{2}\left(\pi \delta_{k} / 2\right) .
$$

We find that $\delta_{k}$ relies only on the geometric ratio $l_{i, j} / l_{k}$, regardless of absolute value of dimensions. Considering a wave with an $E$ field $(\vec{E})$ polarized along the $z$ direction and $\vec{H}$ in the $x-y$ plane, we obtain the relationship between the two first-order magnetic resonances

$$
\begin{gathered}
\omega_{0, x}=\rho \omega_{0, y}, \\
\rho=\sqrt{\frac{\left(\frac{1}{q}\right)^{2}+1+\left(\frac{\delta_{x}}{p}\right)^{2}}{\left(\frac{1}{p}\right)^{2}+1+\left(\frac{\delta_{y}}{q}\right)^{2}},}
\end{gathered}
$$

where $\rho$ is also determined by geometric ratio coefficients $p=l_{x} / l_{z}$ and $q=l_{y} / l_{z}$ (see Appendix A for details). This leads to the first important conclusion that for a fixed set of $\{p, q\}$ (i.e., dielectric cuboids are scaled up or down proportionally in three dimensions), the magnetic resonance frequencies $\omega_{0, x}$ and $\omega_{0, y}$ decrease or increase in a proportional manner.

According to the theory of transformation optics, satisfying a cloaking effect does have requirements in constitutive parameters of the designed metamaterial. With respect to the effective relative permeability of the dielectric cuboid array, it satisfies the Lorentz dispersion relation near the first-order Mie resonance frequency $\omega_{0}$,

$$
\mu(\omega)=1-\frac{\left(\omega_{\mathrm{p}}^{2}-\omega_{0}^{2}\right)}{\left(\omega^{2}-\omega_{0}^{2}+i \gamma \omega\right)},
$$

where $\gamma$ is the collision frequency representing the loss of the cuboids, and $\omega_{\mathrm{p}}$ is the magnetic plasma frequency relating to $\omega_{0}$ in the form [33]

$$
\omega_{\mathrm{p}}=\omega_{0}(1+F)^{1 / 2} .
$$

Note that $F$ is the geometric filling factor, relying on geometric ratio coefficients $p, q$, and $r\left(=a / l_{z}\right)$, i.e.,
$F(p, q, r)$. Assuming $\left|\omega-\omega_{0}\right| \gg \gamma, \mu^{\prime}$ (the real part of $\mu)$, will have a simplified expression

$$
\mu^{\prime} \approx 1-F \omega_{0}^{2} /\left(\omega^{2}-\omega_{0}^{2}\right) .
$$

Then, we can get the expected $\mu$ at the frequency

$$
\omega(\mu)=\omega_{0}[1+F /(1-\mu)]^{1 / 2} .
$$

If there exists a frequency at which $\left\{\mu_{x}, \mu_{y}\right\}$ meets the requirement of the cloak, i.e., $\omega_{x}\left(\bar{\mu}_{x}\right)=\omega_{y}\left(\bar{\mu}_{y}\right)$, the following constraint (again in a complicated form only depending on $p, q$, and $r$ ) needs to hold:

$$
\frac{1+\frac{F_{x}}{1-\bar{\mu}_{x}}}{1+\frac{F_{y}}{1-\bar{\mu}_{y}}}=\frac{1+\frac{1}{q^{2}}+\frac{\delta_{x}^{2}}{p^{2}}}{1+\frac{1}{p^{2}}+\frac{\delta_{y}^{2}}{q^{2}}} .
$$

Therefore, we can make two important statements so far: First, once we conclude $\bar{\mu}_{x}$ and $\bar{\mu}_{y}$ from the theory for the ideal cloak, we can then designate the required values of $p, q$, and $\mathrm{r}$. Second, the fulfillment frequency of $\left\{\bar{\mu}_{x}, \bar{\mu}_{y}\right\}$ can shift higher or lower by scaling down or up the dimensions of the cuboid.

In addition, we also need to pay attention to the other relevant electromagnetic parameter for the cloaking effect, $\epsilon_{z}$, which has the following expression according to Maxwell-Garnett theory [34]:

$$
\epsilon_{z}=\epsilon_{b}+\frac{2 f \epsilon_{b}\left(\epsilon_{a}-\epsilon_{b}\right)}{\left[2 \epsilon_{b}+(1-f)\left(\epsilon_{a}-\epsilon_{b}\right)\right]},
$$

where $\epsilon_{a}$ and $\epsilon_{b}$ are the permittivity of the dielectric cuboids and background material, respectively, and $f=$ $l_{x} l_{y} l_{z} / a^{3}$ is the volumetric filling ratio of the cuboids, which can also be expressed in the form of $p q / r^{3}$. Therefore, $\epsilon_{z}$ does have the same property as $\mu_{x, y}$-it only relies on the geometric ratio coefficients $p, q$, and $r$, rather than on the absolute dimensions of cuboids. On one hand, such a feature for all the relevant electromagnetic parameters $\left\{\mu_{x}, \mu_{y}, \epsilon_{z}\right\}$ makes a dielectric cloak with predesignated dimensions scalable to operate at different frequencies. On the other hand, we show an important relationship between $\epsilon_{z}$ and $\epsilon_{a}$ from the expression aboveas long as the value of $\epsilon_{a}$ is large enough, $\epsilon_{z}$ remains nearly the same, independent of the change of $\epsilon_{a}$. This will be greatly beneficial in simplifying our workflow to realize a frequency-agile cloaking device as shown below.

Based on the above investigations, our proposed all-dielectric metamaterial cloak offers a strategy for changing operational frequencies of the device by directly scaling up or down the predesignated dimensions of Mie particles. However, such a prescription for a cloak is passive, and reconstruction of the device is required to work at expected frequencies. It is highly desirable to realize a frequency-agile 

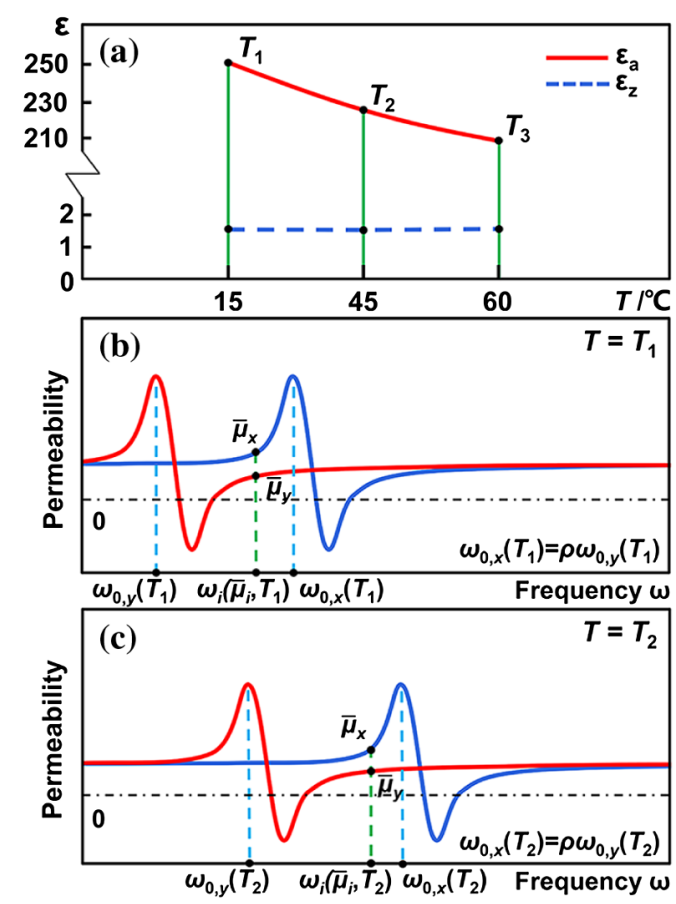

FIG. 2. Mechanism of the temperature-tunable cloak. (a) The temperature dependence of intrinsic permittivity and effective permittivity of the dielectric cuboids. At different temperatures $T_{1}$ and $T_{2}$, the cloak works at different frequencies, and the corresponding characteristic curves are shown in (b) and (c), respectively. When the temperature is changed, the resonance frequencies $\omega_{0, x}$ and $\omega_{0, y}$ are kept proportional, which can be expressed as $\omega_{0, x}=\rho \omega_{0, y}$. Meanwhile, the same property applies to the working frequency $\omega_{i}\left(\bar{\mu}_{i}\right)$ and resonance frequencies $\omega_{0, i}$ in the $i$ direction $(i=x, y)$; i.e., $\omega_{i}\left(\bar{\mu}_{i}\right)=\bar{\rho}_{i} \omega_{0, i}$, in which $\bar{\rho}_{i}$ is the proportionality constant. In other words, the working frequencies in the $x$ and $y$ directions are proportional. The same logic applies when the temperature is changed from $T_{1}$ to $T_{3}$, so even though the permeabilities $\mu_{x}$ and $\mu_{y}$ both change with the temperature, they can still meet the required invisible parameters at another working frequency.

cloak controlled with some external stimuli. We note that the effective electromagnetic parameters can be easily modified by changing the permittivity of the cuboid [35], and it has been demonstrated that the permittivity of the dielectric cuboids can be tuned by changing the temperature [36]. Here, we would like to propose a chameleonlike all-dielectric cloak tuned via temperature. The underlying mechanism (Fig. 2) is essentially led by the intriguing electromagnetic properties of Mie particles. First of all, the intrinsic permittivity of $\mathrm{SrTiO}_{3}$ cuboids is temperature dependent [see Fig. 2(a) and Appendix B for details]. On one hand, for a designed cloak with the required electromagnetic parameters, $\epsilon_{z}$ will stay around $\bar{\epsilon}_{z}$, provided that $\epsilon_{a}$ stays large enough within the changing range of temperature. On the other hand, with respect to the required $\left\{\bar{\mu}_{x}, \bar{\mu}_{y}\right\}$, we found the following property, which is crucial to our temperature-controlled alldielectric cloaking device. Assuming

$$
\omega_{x}\left(\bar{\mu}_{x}, T_{1}\right)=\omega_{y}\left(\bar{\mu}_{y}, T_{1}\right)
$$

where $T_{1}$ represents one temperature point, we have proved that when the temperature changes to $T_{2}$, the resonance frequencies $\omega_{0, x}\left(T_{2}\right)$ and $\omega_{0, y}\left(T_{2}\right)$ are still proportional, i.e.,

$$
\omega_{0, x}\left(T_{2}\right)=\rho \omega_{0, y}\left(T_{2}\right) .
$$

Furthermore, this results in (also see Appendix B for detailed proof)

$$
\omega_{x}\left(\bar{\mu}_{x}, T_{2}\right)=\omega_{y}\left(\bar{\mu}_{y}, T_{2}\right) .
$$

Therefore, for a fixed all-dielectric cloak design, we can achieve the chameleonlike cloaking effect with working frequencies controllable by temperature. Besides the simplicity in the construction of cloaking devices, Mie-cuboidbased metamaterials may provide an effective strategy in realizing frequency-agile cloaks.

\section{REALIZATION OF A CHAMELEONLIKE ALL-DIELECTRIC CLOAK}

To verify the theory above, here we provide the proof-ofconcept simulation (Fig. 3) and experimental (Fig. 4) demonstrations for a temperature-controlled all-dielectric cloak. The $\mathrm{SrTiO}_{3}$ cuboids with permittivity of $250+5 \mathrm{i}$ at temperature $25^{\circ} \mathrm{C}$ were used to construct the rhombic cloak. Via meticulous design, the geometric ratios of the dielectric cuboids can be set as $p=5 / 3, q=2 / 3$, and $r=2$ (see Fig. S4 in Ref. [31]). To make the cloak work at the microwave band, the dimensions of cuboids should be on the millimeter scale and are set as $l_{x}=2.5 \mathrm{~mm}$, $l_{y}=1.0 \mathrm{~mm}, l_{z}=1.5 \mathrm{~mm}$, and $a=3.0 \mathrm{~mm}$, which can achieve the cloak-required anisotropic electromagnetic parameters. The constructed rhombic cloak with side length of $40.00 \mathrm{~mm}$ was composed of 432 dielectric cuboids in total. An aluminum quadrangular was placed at the center as the object to be cloaked, which has a quarter-wave separation to the inner boundaries of the device to approximate the inner edge of the perfect magnetic conductor (PMC) as required.

Figure 3(c) shows the simulated two-dimensional (2D) electric-field distribution without the cloak but only the aluminum quadrangular settled in the optical path. The TE-polarized plane wave impinges upon the device from the left side. As expected, the incident wave is scattered dramatically, resulting in a deep shadow in the forward direction and a strong standing wave to the left. In contrast, at the desired working frequency, the introduction of the cloak significantly decreases the scattering; as shown in Fig. 3(e), the device guides the wave traveling around the inner object, and then the wave is restored in both amplitude and phase upon exiting the cloak on the right. Off the operating frequency, the device can no longer cloak the inner quadrangular. Instead, it behaves like a larger 

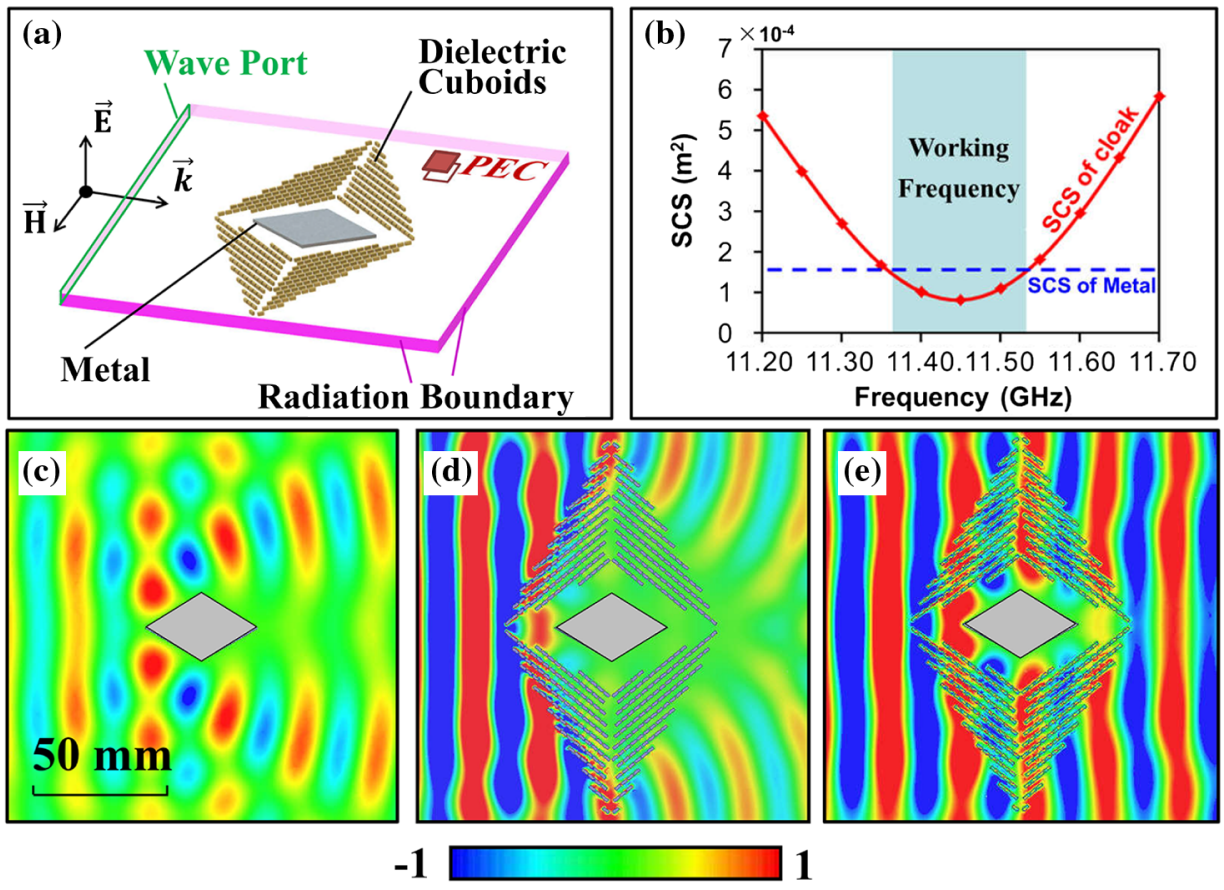

FIG. 3. Full-wave simulation of the designed cloak. (a) Simulated model. (b) Scattering cross sections of the conducting quadrangular with or without the cloak at different frequencies. The calculated electric-field distribution of the conducting quadrangular without the cloak at $11.45 \mathrm{GHz}$ (c), with the cloak at a nonworking frequency of $12.00 \mathrm{GHz}$, (d) and with the cloak at a working frequency of $11.45 \mathrm{GHz}(\mathrm{e})$.

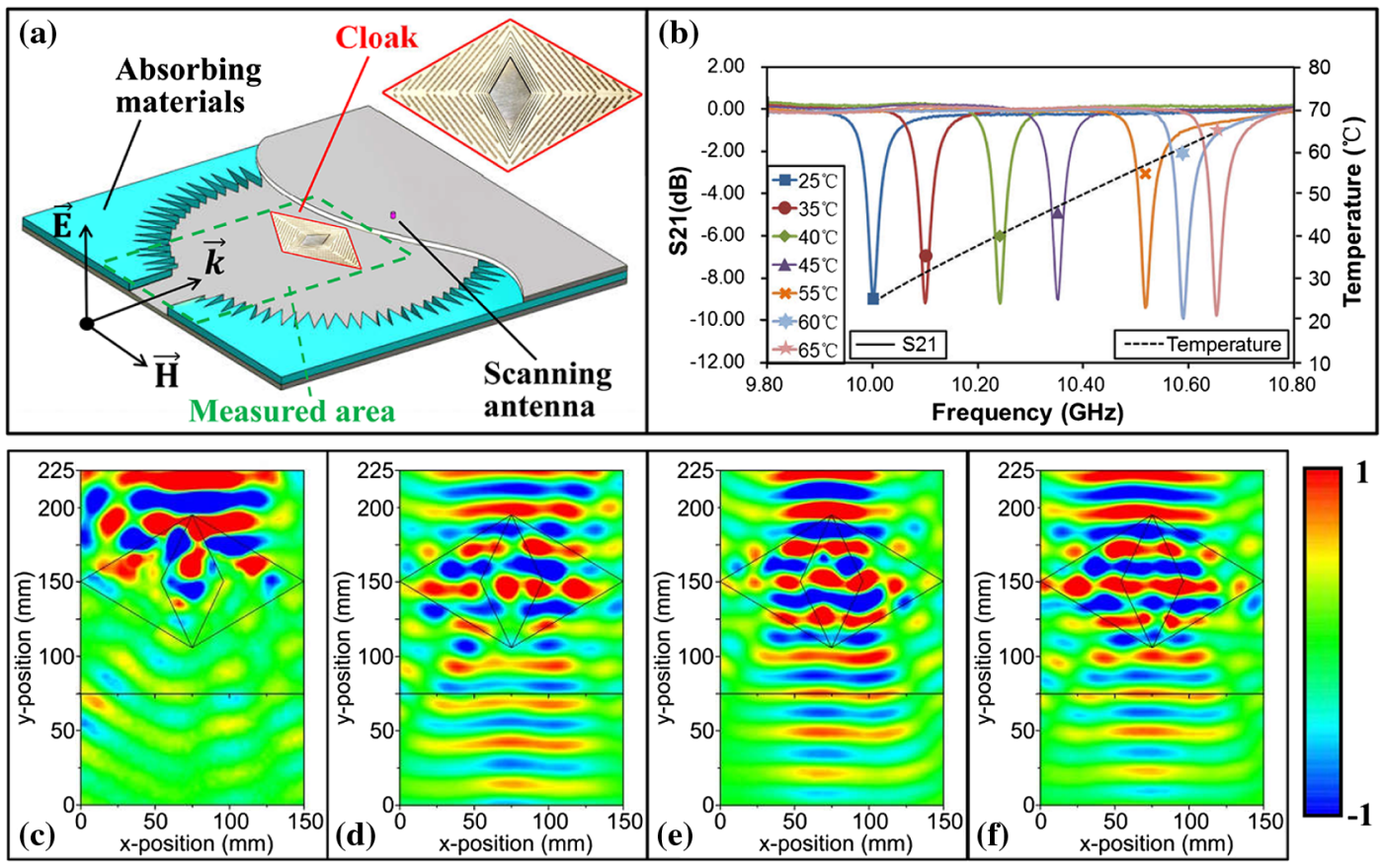

FIG. 4. Experimental measurement of the fabricated cloak. (a) Fabricated chameleonlike cloak and electric-field measurement system. (b) $\mathrm{S}_{21}$ of the dielectric cuboid with $\mathrm{H}$ polarized along the $y$ direction. The frequency of the resonance peak increases with the increase of temperature. Measured electric-field distributions at different temperatures and frequencies: (c) $25^{\circ} \mathrm{C}, 10.00 \mathrm{GHz}$; (d) $15^{\circ} \mathrm{C}, 11.18 \mathrm{GHz}$; (e) $25^{\circ} \mathrm{C}, 11.50 \mathrm{GHz}$; (f) $40^{\circ} \mathrm{C}, 11.72 \mathrm{GHz}$. 
object, which greatly prevents the transmission of the incident wave [Fig. 3(d)]. To quantify the cloaking effect of our constructed device, the scattering cross section (SCS) is introduced for analysis [Fig. 3(b)]. The SCS of the bare aluminum quadrangular is $162.99 \mathrm{~mm}^{2}$, and the application of the cloak decreases the SCS to $81.96 \mathrm{~mm}^{2}$, a reduction of $50 \%$ within the working frequency band; off the working frequencies, the SCS increases dramatically, and it coincides with our numerical demonstration of the field plot. We note that, as a proof-of-concept demonstration, this experiment was implemented to verify our proposed theory, and accordingly, a cloak design with relatively small dimensions was adopted to facilitate the measurements. In this way, the nonideal PMC boundary condition would have a significant impact on the cloaking effect. Nevertheless, the cloaking performance can be greatly improved by optimizing the inner approximate PMC boundary condition and enlarging the device dimensions (see Fig. S5 in Ref. [31]).

To verify the frequency tunability of the cloak, electricfield distributions are measured at different temperatures and shown in Fig. 4 with the microwave incident from the top. Figure 4(a) shows the experimental system. Figures 4(c)-4(f) show the measured $E$-field maps of the cloak at nonworking and working frequencies, respectively. Consistent with the simulations, at a nonworking frequency of $10.00 \mathrm{GHz}$, the incident wave is strongly scattered and reflected at a temperature of $25^{\circ} \mathrm{C}$, as shown in Fig. 4(c), with only a little electromagnetic energy detected at the exiting side of the cloak. In contrast, at the working frequencies of $11.50 \mathrm{GHz}$, the 2D E-field maps of the cloak are shown in Fig. 4(e). The incident radiation is guided around the aluminum quadrangular and eventually restored in both amplitude and phase upon exiting the cloak. Then, what if we change the temperature? As shown in Figs. 4(d) and 4(f), when the temperature is changed to $15^{\circ} \mathrm{C}$ and $40{ }^{\circ} \mathrm{C}$, the working frequency changes to $11.18 \mathrm{GHz}$ and $11.72 \mathrm{GHz}$, respectively. In all three cases of Figs. 4(d)-4(f), the overall cloaking performance is fairly satisfying. The finite energy loss and distortion of the phase fronts of the transmitted wave through the device, especially in the center of the cloak, may be attributed to the intrinsic loss of the dielectric materials and imperfect fabrication of the device. When the temperature increases from $15^{\circ} \mathrm{C}$ to $40^{\circ} \mathrm{C}$, the working frequency shifts from $11.18 \mathrm{GHz}$ to $11.72 \mathrm{GHz}$, correspondingly, covering a band of about $500 \mathrm{MHz}$. It should be noted that, in our proof-ofconcept experiments, the temperature was only changed within a small range limited by the experimental equipment, resulting in the tunable cloaking effect within a narrow band. If the temperature can be changed over a large range, about several hundred degrees, a designed cloaking device is expected to be operational within a fairly broad band. To further confirm the characteristics of temperature and operational frequency, the transmission of a single dielectric cuboid is measured at different temperatures [Fig. 4(b)]. The absorption peak of the cuboids in the $x$ direction increases from $10.00 \mathrm{GHz}$ to $10.65 \mathrm{GHz}$, while the temperature increases from $25^{\circ} \mathrm{C}$ to $65^{\circ} \mathrm{C}$, an obvious indication of the temperature dependence of the cuboid permittivity, which is in agreement with the relationship of Eq. (B1) in Appendix B. Therefore, we successfully experimentally achieved satisfying cloaking performance of an all-dielectric unidirectional cloak with the tunable operational frequency by temperature.

\section{DISCUSSION}

The advantages of the demonstrated cloak are threefold: the universal design method, the simple construction, and most importantly, the frequency-agile invisibility. The proposed design theory dictates a universal and convenient theoretical design strategy: i.e., the dielectric cuboids rather than metallic unit structures can be used to realize the required anisotropic electromagnetic parameters of the cloak, and the different invisible frequency of the cloak can be easily adjusted simply by scaling the sizes of the dielectric cuboids. In this way, redesigning the overall structure of the cloak is not necessary. For example, when the size of the dielectric cuboid is scaled down 10 or 100 times, the invisible frequency proportionally blueshifts from $12.6 \mathrm{GHz}$ to $126 \mathrm{GHz}$ and then to $1.26 \mathrm{THz}$ (Fig. 5), which further confirms our theoretical predictions [Eq. (A16) in Appendix A]. Therefore, the invisible frequency band of the demonstrated dielectric cloak can be easily extended from microwave to $\mathrm{THz}$ frequencies as long as the high permittivity of the cuboid can be achieved at the working frequency. It is known that $\mathrm{Ba}_{0.5} \mathrm{Sr}_{0.5} \mathrm{TiO}_{3}$ possesses a high permittivity of 200 around $1 \mathrm{THz}$ [37], which may serve as a good candidate towards the alldielectric cloak in the $\mathrm{THz}$ regime.

As the cloak is composed of easily constructed dielectric cuboids, the overall assembly of the cloak is achieved with facility. Instead of complicated fabrications of some previously reported cloaks with metallic elements [20], only dielectric cuboids with uniform size are needed to achieve all the required anisotropic electromagnetic parameters for cloaking, so the overall construction of the device would be much easier. Furthermore, the suggested cuboids can also be used in designing two-dimensional cylindrical cloaks with complex anisotropic and inhomogeneous parameters by simply changing their side-length ratios.

As is well known, realizing tunable invisibility is an important but very challenging issue. Our demonstrations here explicitly show that our proposals provide significant progress for frequency-agile cloaking devices. When the permittivity of the cuboid changes from 120 to 400, the invisible frequency will change from $17.00 \mathrm{GHz}$ to $9.00 \mathrm{GHz}$ (see Fig. S6 in Ref. [31]). In addition, the temperaturedependent permittivity is a convenient and efficient tunable method for cloaking. We also theoretically verified that such 

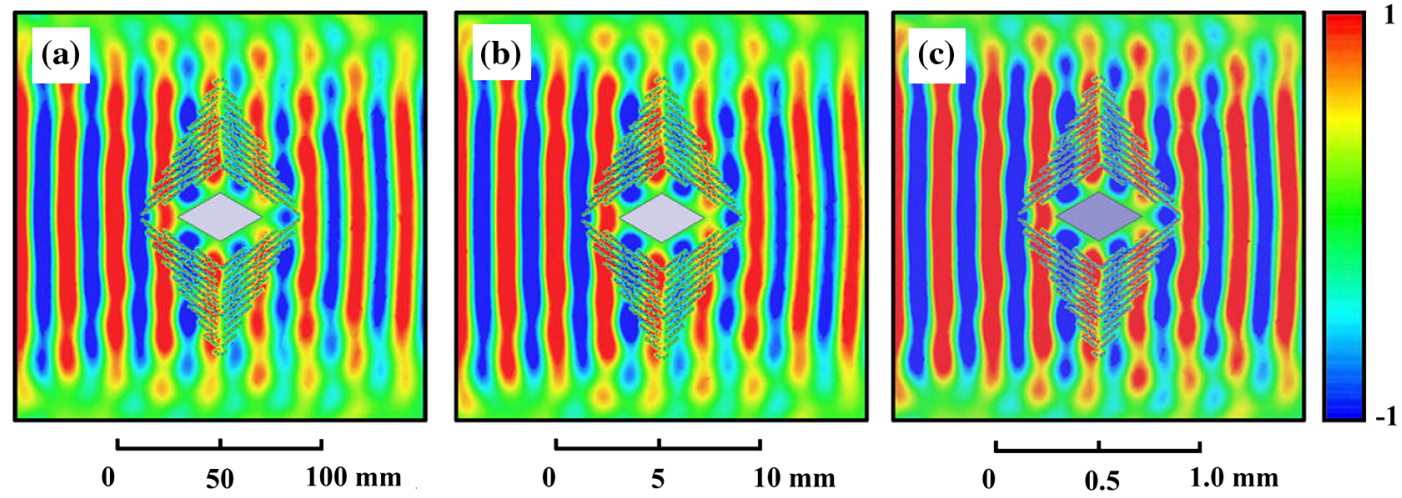

FIG. 5. Numerical demonstration of the frequency-tunable mechanism of the cloak by scaling the dimensions of the cuboids. The permittivity of the cuboids is set to 200 with different sizes. (a) The same size as the cuboids in the experiment, with a working frequency of $12.6 \mathrm{GHz}$. (b) Scaling down the size to $1 / 10$, with a working frequency of $126 \mathrm{GHz}$. (c) Scaling down the size to $1 / 100$, with a working frequency of $1.26 \mathrm{THz}$.

a temperature-tunable mechanism can be applied to the twodimensional all-dielectric cylindrical cloak suggested by Gaillot et al. [38] (see Appendix C for details).

It should be noted that in our experiments the distance between the metal object and the cloak remains fixed as the frequency is tuned, which may limit the tuning bandwidth, in practice. However, in our proof-of-concept experiments, we aim to demonstrate the proposed theory and only change the temperature in a small range, resulting in a shift of $0.54 \mathrm{GHz}$ of the working frequency. Therefore, the fixed distance may have less impact on the cloaking effect in our experiments. In addition, we also propose an advanced solution for special cases in which drastic response tuning is required (see Appendix D for details).

As the coordinate transformation methods work similarly, our proposed all-dielectric unidirectional cloak can potentially work as a temperature-controlled carpet cloak as well, which can conceal an object on the ground plane. By keeping the upper part of the unidirectional cloak while removing the lower part and setting the inner boundary as a perfect electric conductor, the device can be readily transformed to a perfect carpet cloak (see Fig. S7 in Ref. [31]). It should be noted that to avoid extremely high or low refractive indices, the volume of the reported carpet cloaks is generally much larger than the concealed object. In addition, to prevent the necessary index of less than 1 , the reported cloaks are generally embedded in a dielectric prism, which would inevitably introduce an additional phase delay in the reflected light and eventually cause the optical cloak's undesirable visibility. Instead, our proposed carpet cloak, which is much smaller in size and easier in fabrication, provides a perfect solution for these above-mentioned issues, rendering the object invisible in the air without introducing unnecessary phase shifts.

\section{CONCLUSION}

In summary, we have proposed a simple design theory for the unidirectional all-dielectric temperature-tunable transformation-optics-based cloak and experimentally demonstrated a prototype device. More importantly, we proposed a mechanism for designing all-dielectric cloaks with the operational frequency adjustable either by scaling the overall dimension of the building blocks or, more easily, by applying external stimuli, such as temperature. Our design strategy not only works for unidirectional cloaks, but it can also be readily applied to 2D cylindrical cloaks with more complicated gradient electromagnetic parameters. Furthermore, the invisibility in the terahertz regime may also be achievable with the proposed design theory. This work paves an easy way to design and construct tunable transformation-optics cloaks and draws an important step on the path towards the practical application of the invisible cloak.

\section{ACKNOWLEDGMENTS}

This work is supported by the National Natural Science Foundation of China (Grants No. 61275176, No. 515 75297, No. 11372248, No. 61505164, No. 51532004, and No. 51323006), the Program for New Century Excellent Talents in University (Grant No. NCET-130337), the Science and Technology Plan of Shenzhen City (Grant No. JCYJ20160301154309393), the Chinese State Key Laboratory of Tribology, and State Key Laboratory of Fluid Power and Mechatronic Systems (Zhejiang University, Grant No. GZKF-201509). Work at Ames Laboratory was partially supported by the U.S. Department of Energy, Office of Basic Energy Science, Division of Materials Sciences and Engineering under Contract No. DE-AC02-07CH11358, the U.S. Office of Naval Research Award No. N00014-14-1-0474 (simulations), and the European Research Council under the ERC Advanced Grant No. 320081 (PHOTOMETA) supported work (theory) at FORTH. The authors gratefully acknowledge discussions with Dr. L. Kang, Dr. J. B. Sun, and Dr. P. Cheng.

R. P. and Z.X. contributed equally to this work. 


\section{APPENDIX A: ANISOTROPIC ELECTROMAGNETIC PARAMETERS}

For an incident-plane electromagnetic wave, the first Mie magnetic-dipole resonance frequency along the $k$ direction is [32]

$$
\omega_{0, k}=\frac{\pi c_{0}}{\sqrt{\epsilon_{a} \mu_{a}}} \cdot \sqrt{\left(\frac{1}{l_{i}}\right)^{2}+\left(\frac{1}{l_{j}}\right)^{2}+\left(\frac{\delta}{l_{k}}\right)^{2}},
$$

where $c_{0}$ is the speed of light in vacuum, $\epsilon_{a}$ and $\mu_{a}$ are the relative permittivity and permeability of the cuboids, $l_{i}$ and $l_{j}$ represent the dimensions of the rectangular cross section ( $i$-j plane) perpendicular to the magnetic vector $\vec{H}, l_{k}$ is the cuboid size along $\vec{H}$, and $\delta(0<\delta<1)$ denotes a fraction of a half-wavelength in the $l_{k}$ direction and is subject to the following constraint:

$$
\left(\frac{\delta_{k}}{l_{k}}\right)^{2}=\left(\epsilon_{a}-1\right) \frac{\omega_{0, k}^{2}}{\pi^{2} c^{2}} \cos ^{2}\left(\frac{\pi \delta_{k}}{2}\right) .
$$

Thus, for a wave polarized along the $z$ direction and magnetic vectors along the $x$ or $y$ directions, the first-order magnetic resonances are as follows:

$$
\begin{aligned}
& \omega_{0, x}=\frac{\pi c_{0}}{\sqrt{\epsilon_{a} \mu_{a}}} \cdot \sqrt{\left(\frac{1}{l_{y}}\right)^{2}+\left(\frac{1}{l_{z}}\right)^{2}+\left(\frac{\delta_{x}}{l_{x}}\right)^{2}}, \\
& \omega_{0, y}=\frac{\pi c_{0}}{\sqrt{\epsilon_{a} \mu_{a}}} \cdot \sqrt{\left(\frac{1}{l_{z}}\right)^{2}+\left(\frac{1}{l_{x}}\right)^{2}+\left(\frac{\delta_{y}}{l_{y}}\right)^{2}},
\end{aligned}
$$

where $l_{x}, l_{y}$, and $l_{z}$ are the side lengths of the cuboid. Thus, the relationship between $\omega_{0, x}$ and $\omega_{0, y}$ satisfies

$$
\begin{gathered}
\omega_{0, x}=\rho \omega_{0, y}, \\
\rho=\sqrt{\frac{\left(\frac{1}{q}\right)^{2}+1+\left(\frac{\delta_{x}}{p}\right)^{2}}{\left(\frac{1}{p}\right)^{2}+1+\left(\frac{\delta_{y}}{q}\right)^{2}},}
\end{gathered}
$$

where $p$ and $q$ are defined as the ratio coefficients, namely, $p=l_{x} / l_{z}$ and $q=l_{y} / l_{z}$. Substituting Eq. (A1) into Eq. (A2), it is easy to conclude that as long as the ratios of the geometric dimensions $p$ and $q$ are fixed, $\delta_{k}$ remains unchanged, regardless of the absolute value of the geometric dimensions, and this regularity also applies to the ratio of the resonance frequencies $\rho$ from Eq. (A6).

The effective relative permeability of the dielectric cuboid array satisfies the Lorentz dispersion model near the first-order Mie resonance frequency $\omega_{0}$ :

$$
\mu(\omega)=1-\frac{\omega_{p}^{2}-\omega_{0}^{2}}{\omega^{2}-\omega_{0}^{2}+i \gamma \omega},
$$

where $\omega_{p}$ and $\omega_{0}$ are the magnetic plasma frequency and the magnetic resonance frequency, respectively, and $\gamma$ is the quantity related to the dielectric loss of the cuboids. It is known that $\omega_{p}$ is proportional to $\omega_{0}$, and the ratio satisfies [33]

$$
\omega_{p}=\sqrt{1+F} \omega_{0},
$$

where $F$ is the filling factor related to the geometric parameters of the cuboids. Define $r$ as the ratio coefficient of the characteristic dimension of the cuboid's array $a$, and the side length $l_{z}$, namely, $r=a / l_{z}$. Here, $F$ can be expressed as

$$
F=F(p, q, r) \text {. }
$$

Generally, $\gamma$ is a higher-order infinitesimal quantity compared to $\omega_{0}$, and when the restriction $\left|\omega-\omega_{0}\right| \gg \gamma$ is satisfied, we can get the following approximation by combining the equations above:

$$
\mu \prime(\omega) \approx 1-\frac{F \omega_{0}^{2}}{\omega^{2}-\omega_{0}^{2}},
$$

where $\mu^{\prime}$ is the real part of $\mu$. The working frequencies $\omega$ in the $x$ and $y$ directions can be expressed as

$$
\begin{gathered}
\omega_{x}\left(\mu_{x}\right)=\rho_{x} \omega_{0, x}, \\
\omega_{y}\left(\mu_{y}\right)=\rho_{y} \omega_{0, y}, \\
\rho_{x}=\sqrt{1+\frac{F_{x}}{1-\mu_{x}}}, \quad \rho_{y}=\sqrt{1+\frac{F_{y}}{1-\mu_{y}}},
\end{gathered}
$$

where $\mu_{x}$ and $\mu_{y}$ are the anisotropic permeabilities in the $x$ and $y$ directions, respectively, and $F_{x}$ and $F_{y}$ are the anisotropic filling factors. If there exists a frequency $\omega$ that makes the real part of the permeabilities $\mu_{x}$ and $\mu_{y}$ meet the theoretically desired values $\bar{\mu}_{x}$ and $\bar{\mu}_{y}$ of an ideal cloak, the cloak can work, i.e.,

$$
\omega_{x}\left(\bar{\mu}_{x}\right)=\omega_{y}\left(\bar{\mu}_{y}\right) .
$$

Thus,

$$
\bar{\rho}_{y}=\rho \bar{\rho}_{x}
$$

In other words,

$$
\frac{1+\frac{F_{x}}{1-\bar{\mu}_{x}}}{1+\frac{F_{y}}{1-\bar{\mu}_{y}}}=\frac{\left(\frac{1}{q}\right)^{2}+1+\left(\frac{\delta_{x}}{p}\right)^{2}}{\left(\frac{1}{p}\right)^{2}+1+\left(\frac{\delta_{y}}{q}\right)^{2}} .
$$

Therefore, once we conclude $\bar{\mu}_{x}$ and $\bar{\mu}_{y}$ from the theory for an ideal cloak and the calculated values are between the minimum and maximum values $\left(\mu_{\min }\right.$ and $\left.\mu_{\max }\right)$, which are prescribed by the frequency characteristic curve of the dielectric cuboids, we can then always designate certain values of $p, q$, and $r$ to validate Eq. (A15). Equation (A10), inferred that $\mu_{\min }$ and $\mu_{\max }$ are only determined by the filling factor $F$. In addition, Eq. (A16) shows that the equality 
depends on the geometric ratios $p, q$, and $r$ rather than the geometric dimensions $l_{x}, l_{y}, l_{z}$, and $a$. In other words, as long as $p, q$, and $r$ are kept constant and the cloak can work at a certain frequency, the working frequency can then shift higher or lower by simply scaling down or up the dimensions of the cuboid.

\section{APPENDIX B: MECHANISM OF THE TEMPERATURE-TUNABLE CLOAK}

Generally, there are two types of strategies to change the working frequency of the proposed all-dielectric cloak, i.e., by varying the dimension of the device or by changing the permittivity of the cuboids [Fig. 1(c)]. For the dimensiontunable case, which is passive and unadjustable, the cloak must be reconstructed to make the effective parameters meet the requirements. Here, we consider the permittivitytunable method and verify it experimentally. We note that, although the effective electromagnetic parameters can be easily modified by changing the permittivity of the cuboid [35], it is challenging to compensate for variations of the electromagnetic parameters in different directions while satisfying the required parameters at the new frequency.

It has been demonstrated that the permittivity of the dielectric cuboids can be tuned by changing the temperature, which implies that the dielectric cuboids can potentially be used to realize the anisotropic electromagnetic parameters of the proposed cloak. Ferroelectric materials are widely employed in tunable devices at the microwave regime because of their ideal characteristics of high permittivity, low loss, tunability, etc. For example, $\mathrm{SrTiO}_{3}$ is a typical ferroelectric material, and the relation between its permittivity and temperature satisfies [36]

$$
\left(1 / \epsilon^{2}\right)(\partial \epsilon / \partial T)=-1 / c,
$$

where $c=68000 \mathrm{~K}$ is the Curie constant, as shown in Fig. 2(a). Thus, we rewrite Eq. (A14) as function of temperature $T_{1}$ as follows:

$$
\omega_{x}\left(\bar{\mu}_{x}, T_{1}\right)=\omega_{y}\left(\bar{\mu}_{y}, T_{1}\right) .
$$

Accordingly, changing the temperature to $T_{2}$ will change the intrinsic permittivity and the frequency response curve of the dielectric cuboids (Fig. 2). From Eq. (A5), it can be inferred that the resonance frequencies in the $x$ and $y$ directions are still proportional:

$$
\omega_{0, x}\left(T_{2}\right)=\rho \omega_{0, y}\left(T_{2}\right) .
$$

Meanwhile, the same property applies to the working frequency $\omega_{i}\left(\mu_{i}\right)$ and resonance frequency $\omega_{0, i}$ in the $i$ direction $(i=x, y)$ according to Eqs. (A11) and (A12), i.e.,

$$
\begin{aligned}
& \omega_{x}\left(\bar{\mu}_{x}, T_{2}\right)=\bar{\rho}_{x} \omega_{0, x}\left(T_{2}\right), \\
& \omega_{y}\left(\bar{\mu}_{y}, T_{2}\right)=\bar{\rho}_{y} \omega_{0, y}\left(T_{2}\right) .
\end{aligned}
$$

We then conclude the relationship between $\omega_{x}\left(\bar{\mu}_{x}, T_{2}\right)$ and $\omega_{y}\left(\bar{\mu}_{y}, T_{2}\right)$ by substituting Eqs. (A15) and (B3) into Eqs. (B4) and (B5); that is,

$$
\omega_{x}\left(\bar{\mu}_{x}, T_{2}\right)=\omega_{y}\left(\bar{\mu}_{y}, T_{2}\right) .
$$

Therefore, after the temperature changes from $T_{1}$ to $T_{2}$, another frequency appears at which the cuboids can realize the required permeability $\bar{\mu}_{x}$ and $\bar{\mu}_{y}$ in the two directions. In addition, the effective permittivity $\epsilon_{z}$ remains mostly constant with changes in temperature as long as $\epsilon_{a}>100$, as shown in the main text. Though there may be dielectric dispersion for the ferroelectrics of interest here in the $\mathrm{THz}$ region, it is still possible to eliminate the mismatch between the actual and the required anisotropy of the cuboids by a fine adjustment of the temperature. In other words, the cuboids cannot achieve the required two anisotropic permeabilities at the excepted temperature, but we can further adjust the temperature and change the permittivity to offset the impact of the dielectric relaxation. We emphasize that this flexibility in manipulation of electromagnetic anisotropy is the core contribution of our work. Consequently, the proposed all-dielectric cloak is a temperature-controlled chameleonlike cloak, where the working frequency is indeed tunable by external stimuli.

In addition, our theory shows that the tunability of the cloak only depends on the dielectric constant of the cuboid, and the demonstration was successful and convincing. Considering the fact that the dielectric constant of ceramics can be regulated by other external stimuli, such as the electric field [39] and the stress field [40], etc., we expect that the temporal response of the proposed active cloak can be much better, though it is beyond the scope of this study.

\section{APPENDIX C: TEMPERATURE-CONTROLLED TWO-DIMENSIONAL CYLINDER CLOAK}

Temperature-controlled permittivity is a convenient tunable method for cloaking, and such temperature-tunable mechanisms can also be applied to the two-dimensional cylindrical cloak [38]. To achieve the continuous inhomogeneous electromagnetic parameters required by the cylindrical cloak, the cloak mediums are discretized into $n$ layers, and each layer has a homogeneous medium. Define $\omega_{0, i r}$ and $\omega_{0, i \theta}$ as the first-order magnetic resonance frequencies of the cuboids in layer $i$ for the wave polarized along the $z$ direction and the magnetic vector along the $r$ or $\theta$ direction:

$$
\begin{aligned}
& \omega_{0, i r}=\frac{\pi c_{0}}{\sqrt{\epsilon_{a} \mu_{a}}} \cdot \sqrt{\left(\frac{1}{l_{i \theta}}\right)^{2}+\left(\frac{1}{l_{i z}}\right)^{2}+\left(\frac{\delta_{i r}}{l_{i r}}\right)^{2}}, \\
& \omega_{0, i \theta}=\frac{\pi c_{0}}{\sqrt{\epsilon_{a} \mu_{a}}} \cdot \sqrt{\left(\frac{1}{l_{i z}}\right)^{2}+\left(\frac{1}{l_{i r}}\right)^{2}+\left(\frac{\delta_{i \theta}}{l_{i \theta}}\right)^{2}},
\end{aligned}
$$


where $l_{i r}, l_{i \theta}$, and $l_{i z}$ are the corresponding side lengths of the cuboid in layer $i$, and $\delta_{i r}$ and $\delta_{i \theta}$ denote a fraction of half-wavelength in the $r$ and $\theta$ directions, respectively, and are subject to the analogous constraint of Eq. (A2) above. Thus, the relationship between $\omega_{0, i r}, \omega_{0, i \theta}$, and $\omega_{0,1 r}$ satisfies

$$
\begin{gathered}
\omega_{0,1 r}=\rho_{0, i r} \omega_{0, i r}, \\
\omega_{0,1 r}=\rho_{0, i \theta} \omega_{0, i \theta}, \\
\rho_{0, i r}=\sqrt{\frac{\left(\frac{1}{l_{1 \theta}}\right)^{2}+\left(\frac{1}{l_{1 z}}\right)^{2}+\left(\frac{\delta_{1 r}}{l_{1 r}}\right)^{2}}{\left(\frac{1}{l_{i \theta}}\right)^{2}+\left(\frac{1}{l_{i z}}\right)^{2}+\left(\frac{\delta_{i r}}{l_{i r}}\right)^{2}},} \\
\rho_{0, i \theta}=\sqrt{\frac{\left(\frac{1}{l_{1 \theta}}\right)^{2}+\left(\frac{1}{l_{1 z}}\right)^{2}+\left(\frac{\delta_{1 r}}{l_{1 r}}\right)^{2}}{\left(\frac{1}{l_{i z}}\right)^{2}+\left(\frac{1}{l_{i r}}\right)^{2}+\left(\frac{\delta_{i \theta}}{l_{i \theta}}\right)^{2}} .}
\end{gathered}
$$

Define

$$
p_{i}=\frac{l_{i r}}{l_{1 z}}, \quad q_{i}=\frac{l_{i \theta}}{l_{1 z}}, \quad r_{i}=\frac{l_{i z}}{l_{1 z}} .
$$

Correspondingly, $\rho_{0, i r}$ and $\rho_{0, i \theta}$ can be written as

$$
\begin{aligned}
& \rho_{0, i r}=\sqrt{\frac{\left(\frac{1}{q_{1}}\right)^{2}+1+\left(\frac{\delta_{1 r}}{p_{1}}\right)^{2}}{\left(\frac{1}{q_{i}}\right)^{2}+\left(\frac{1}{r_{i}}\right)^{2}+\left(\frac{\delta_{i r}}{p_{i}}\right)^{2}},} \\
& \rho_{0, i \theta}=\sqrt{\frac{\left(\frac{1}{q_{1}}\right)^{2}+1+\left(\frac{\delta_{1 r}}{p_{1}}\right)^{2}}{\left(\frac{1}{r_{i}}\right)^{2}+\left(\frac{1}{p_{i}}\right)^{2}+\left(\frac{\delta_{i q}}{q_{i}}\right)^{2}} .}
\end{aligned}
$$

The effective relative permeability of the dielectric cuboids array in layer $i$ satisfies the Lorentz dispersion model near the first-order Mie resonance

$$
\mu_{i}(\omega)=1-\frac{\omega_{p, i}^{2}-\omega_{0, i}^{2}}{\omega^{2}-\omega_{0, i}^{2}+i \gamma_{i} \omega},
$$

where $\omega_{p, i}$ and $\omega_{0, i}$ are the corresponding magnetic plasma frequency and magnetic resonance frequency, and $\gamma_{i}$ is the quantity related to the dielectric loss of cuboids in layer $i$. Define $\omega_{p, i r}$ and $\omega_{p, i \theta}$ as the magnetic plasma frequency of the cuboids in layer $i$ for the wave with magnetic vector along the $r$ or $\theta$ direction; the relationship between the magnetic plasma frequency and the magnetic resonance frequency satisfies

$$
\begin{aligned}
& \omega_{p, i r}=\sqrt{1+F_{i r}} \omega_{0, i r}, \\
& \omega_{p, i \theta}=\sqrt{1+F_{i \theta}} \omega_{0, i \theta},
\end{aligned}
$$

where $F_{i r}$ and $F_{i \theta}$ are the corresponding filling factors in layer $i$. Using the approximation in the main text, the working frequencies $\omega_{i r}$ and $\omega_{i \theta}$ in two directions $r$ and $\theta$ in layer $i$ can be expressed as

$$
\begin{gathered}
\omega_{i r}\left(\mu_{i r}\right)=\rho_{i r} \omega_{0, i r}, \\
\omega_{i \theta}\left(\mu_{i \theta}\right)=\rho_{i \theta} \omega_{0, i \theta}, \\
\rho_{i r}=\sqrt{1+\frac{F_{i r}}{1-\mu_{i r}}}, \quad \rho_{\theta}=\sqrt{1+\frac{F_{i \theta}}{1-\mu_{i \theta}}} .
\end{gathered}
$$

If there exists a frequency $\omega$ to make the real part of the permeabilities $\mu_{i r}$ and $\mu_{i \theta}$ meet the requirement of an ideal cylinder cloak $\bar{\mu}_{i r}$ and $\bar{\mu}_{i \theta}$, then the cloak can work, i.e.,

$$
\begin{aligned}
\omega_{1 r}\left(\bar{\mu}_{1 r}\right) & =\omega_{1 \theta}\left(\bar{\mu}_{1 \theta}\right)=\cdots=\omega_{i r}\left(\bar{\mu}_{i r}\right)=\omega_{i \theta}\left(\bar{\mu}_{i \theta}\right) \\
& =\cdots=\omega_{n r}\left(\bar{\mu}_{n r}\right)=\omega_{n \theta}\left(\bar{\mu}_{n \theta}\right) .
\end{aligned}
$$

Considering Eqs. (C3), (C4), (C13), and (C14), Eq. (C16) can be written as

$\bar{\rho}_{1 r}=\frac{\bar{\rho}_{1 \theta}}{\rho_{0,1 \theta}}=\cdots=\frac{\bar{\rho}_{1 r}}{\rho_{0, i r}}=\frac{\bar{\rho}_{i \theta}}{\rho_{0, i \theta}}=\cdots=\frac{\bar{\rho}_{n r}}{\rho_{0, n r}}=\frac{\bar{\rho}_{n \theta}}{\rho_{0, n \theta}}$.

It is known that the dielectric cuboids can be used to realize the anisotropic electromagnetic parameters of the cylinder cloak as theoretically verified above; thus, Eq. (C16) at the temperature $T_{1}$ can be rewritten as

$$
\begin{aligned}
\omega_{1 r}\left(\bar{\mu}_{1 r}, T_{1}\right) & =\omega_{1 \theta}\left(\bar{\mu}_{1 \theta}, T_{1}\right)=\cdots=\omega_{i r}\left(\bar{\mu}_{i r}, T_{1}\right) \\
& =\omega_{i \theta}\left(\bar{\mu}_{i \theta}, T_{1}\right)=\cdots=\omega_{n r}\left(\bar{\mu}_{n r}, T_{1}\right) \\
& =\omega_{n \theta}\left(\bar{\mu}_{n \vartheta}, T_{1}\right) .
\end{aligned}
$$

Changing the temperature to $T_{2}$, the intrinsic permittivity and frequency response curve of the dielectric cuboids will change as well. From Eqs. (C3) and (C4), the resonance frequencies in the $r$ and $\theta$ directions in each layer are still proportional:

$$
\begin{aligned}
& \omega_{0,1 r}\left(T_{2}\right)=\rho_{0, i r} \omega_{0, i r}\left(T_{2}\right), \\
& \omega_{0,1 r}\left(T_{2}\right)=\rho_{0, i \theta} \omega_{0, i \theta}\left(T_{2}\right) .
\end{aligned}
$$

Meanwhile, the same property applies to the working frequencies and resonance frequencies in each layer according to Eqs. (C13) and (C14), i.e.,

$$
\begin{aligned}
& \omega_{i r}\left(\mu_{i r}, T_{2}\right)=\bar{\rho}_{i r} \omega_{0, i r}\left(T_{2}\right), \\
& \omega_{i \theta}\left(\mu_{i \theta}, T_{2}\right)=\bar{\rho}_{i \theta} \omega_{0, i \theta}\left(T_{2}\right) .
\end{aligned}
$$

We then conclude the relationship by substituting Eqs. (C17), (C19), and (C20) into Eqs. (C21) and (C22), that is, 


$$
\begin{aligned}
\omega_{1 r}\left(\bar{\mu}_{1 r}, T_{2}\right) & =\omega_{1 \theta}\left(\bar{\mu}_{1 \theta}, T_{2}\right)=\cdots=\omega_{i r}\left(\bar{\mu}_{i r}, T_{2}\right) \\
& =\omega_{i \theta}\left(\bar{\mu}_{i \theta}, T_{2}\right)=\cdots=\omega_{n r}\left(\bar{\mu}_{n r}, T_{2}\right) \\
& =\omega_{n \theta}\left(\bar{\mu}_{n \theta}, T_{2}\right) .
\end{aligned}
$$

Therefore, for a temperature change from $T_{1}$ to $T_{2}$, there exists another frequency at which the cuboids can realize the required permeability $\bar{\mu}_{i r}$ and $\bar{\mu}_{i \theta}$ for every layer $i$. Additionally, the effective permittivity $\epsilon_{i z}$ nearly remains constant with the changes of the temperature. Therefore, such a temperature-tunable mechanism can also be applied to the two-dimensional all-dielectric cylindrical cloak, making it frequency tunable.

\section{APPENDIX D: TEMPERATURE-CONTROLLED PERFECT MAGNETIC CONDUCTOR}

It should be noted that, for the proposed unidirectional cloak, the distance between the metal object and the cloak remains fixed as the frequency is tuned, which may limit the tuning bandwidth, in practice. Here, based on the fact that materials with high permeability reproduce a good equivalent to $\mathrm{PMC}$, we propose an advanced solution to this problem by exploring dielectric-cuboids-enabled approximate-PMC boundary conditions. Simulations verified that the relative SCS of a cloak system with an approximate PMC inner boundary enabled by high-permeability (about 40) material can be as low as $8 \%$. Consequently, dielectric cuboids that undergo high effective permeability near the resonance should be able to provide PMC boundary conditions. In particular, this can be achieved by inserting a layer of well-tailored dielectric cubes whose resonance overlaps with the cloak's working frequency at the inner cloak boundary. A close examination reveals that this method provides multiple benefits. Such an approximating PMC boundary condition can always be satisfied with a changed temperature, and the resonant frequency stays the same as the cloak working frequency all the time. The theoretical derivation is as follows. Similar to the derivations in the Appendix A, we can get the relationship between the magnetic resonance of the cloak cuboids and the approximating PMC cubes as

$$
\omega_{0, i}=\rho_{i, \mathrm{PMC}} \omega_{0, \mathrm{PMC}},
$$

where $\omega_{0, i}$ is the magnetic resonant frequency in the $i$ directions $(i=x, y)$ of the cloak cuboids, $\omega_{0, \mathrm{PMC}}$ is the magnetic resonant frequency of the approximating PMC cubes, and $\rho_{i, \mathrm{PMC}}$ is the proportionality constant related to the geometric parameters of the cuboids and the cubes. In addition, we have concluded that

$$
\omega_{i}\left(\bar{\mu}_{i}\right)=\bar{\rho}_{i} \omega_{0, i},
$$

where $\omega_{i}\left(\bar{\mu}_{i}\right)$ is the working frequency, $\bar{\mu}_{i}$ is the theoretically calculated permeability of the cloak from transformation optics theory, and $\rho_{i}$ is a proportionality constant related to the permeability $\bar{\mu}_{i}$ and the geometric parameters of the cuboids. Design the size of the cubes so that the resonant frequency equals the cloak working frequency, e.g.,

$$
\omega_{i}\left(\bar{\mu}_{i}\right)=\omega_{0, \mathrm{PMC}} .
$$

Substituting Eqs. (D1) and (D2) into Eq. (D3), we obtain

$$
\bar{\rho}_{i} \rho_{i, \mathrm{PMC}}=1 .
$$

Assuming the temperature is $T_{1}$, we rewrite Eq. (D3) as a function of temperature $T_{1}$ as follows:

$$
\omega_{i}\left(\bar{\mu}_{i}, T_{1}\right)=\omega_{0, \mathrm{PMC}}\left(T_{1}\right) .
$$

Accordingly, changing the temperature to $T_{2}$ will change the intrinsic permittivity and the frequency response curve of the dielectric cuboids. From Eq. (D1), it can be inferred that the resonant frequencies $\omega_{0, i}$ and $\omega_{0, \mathrm{PMC}}$ are still proportional:

$$
\omega_{0, i}\left(T_{2}\right)=\rho_{i, \mathrm{PMC}} \omega_{0, \mathrm{PMC}}\left(T_{2}\right) .
$$

Meanwhile, the same property applies to the working frequency $\omega_{i}\left(\mu_{i}\right)$ and resonance frequency $\omega_{0, i}$ according to Eq. (D2), i.e.,

$$
\omega_{i}\left(\bar{\mu}_{i}, T_{2}\right)=\bar{\rho}_{i} \omega_{0, i}\left(T_{2}\right) .
$$

We then conclude the relationship between $\omega_{i}\left(\bar{\mu}_{i}, T_{2}\right)$ and $\omega_{0, \mathrm{PMC}}\left(T_{2}\right)$ by substituting Eqs. (D4) and (D6) into Eq. (D7), that is,

$$
\omega_{i}\left(\bar{\mu}_{i}, T_{2}\right)=\omega_{0, \mathrm{PMC}}\left(T_{2}\right) .
$$

Therefore, we have proved that after the temperature changes from $T_{1}$ to $T_{2}$, the cloak and the approximating PMC can both work at another frequency. The approximate PMC based on dielectric cubes can perfectly solve the problem, and further research should be carried out in the future.

[1] T. Ergin, N. Stenger, P. Brenner, J. B. Pendry, and M. Wegener, Three-Dimensional Invisibility Cloak at Optical Wavelengths, Science 328, 337 (2010).

[2] X. Ni, Z. J. Wong, M. Mrejen, Y. Wang, and X. Zhang, An Ultrathin Invisibility Skin Cloak for Visible Light, Science 349, 1310 (2015).

[3] J. B. Pendry, D. Schurig, and D. R. Smith, Controlling Electromagnetic Fields, Science 312, 1780 (2006).

[4] U. Leonhardt, Optical Conformal Mapping, Science 312 , 1777 (2006).

[5] D. Schurig, J. J. Mock, B. J. Justice, S. A. Cummer, J. B. Pendry, A. F. Starr, and D. R. Smith, Metamaterial Electromagnetic Cloak at Microwave Frequencies, Science 314, 977 (2006). 
[6] W. Cai, U. K. Chettiar, A. V. Kildishev, and V. M. Shalaev, Optical Cloaking with Metamaterials, Nat. Photonics 1, 224 (2007).

[7] S. Xi, H. Chen, B. Wu, and J. A. Kong, One-Directional Perfect Cloak Created with Homogeneous Material, IEEE Microwave Wireless Compon. Lett. 19, 131 (2009).

[8] R. F. Wang, Z. L. Mei, X. Y. Yang, X. Ma, and T. J. Cui, Switchable Invisibility Cloak, Anticloak, Transparent Cloak, Superscatterer, and Illusion for the Laplace Equation, Phys. Rev. B 89, 165108 (2014).

[9] L. Zigoneanu, B. Popa, and S. A. Cummer, ThreeDimensional Broadband Omnidirectional Acoustic Ground Cloak, Nat. Mater. 13, 352 (2014).

[10] H. Xu, X. Shi, F. Gao, H. Sun, and B. Zhang, Ultrathin Three-Dimensional Thermal Cloak, Phys. Rev. Lett. 112, 054301 (2014).

[11] T. Han, X. Bai, D. Gao, J. T. L. Thong, B. Li, and C. W. Qiu, Experimental Demonstration of a Bilayer Thermal Cloak, Phys. Rev. Lett. 112, 054302 (2014).

[12] G. W. Milton, M. Briane, and J. R. Willis, On Cloaking for Elasticity and Physical Equations with a Transformation Invariant Form, New J. Phys. 8, 248 (2006).

[13] J. Y. Lee and R. Lee, Hiding the Interior Region of Core-Shell Nanoparticles with Quantum Invisible Cloaks, Phys. Rev. B 89, 155425 (2014).

[14] Y. Ma, Y. Liu, M. Raza, Y. Wang, and S. He, Experimental Demonstration of a Multiphysics Cloak: Manipulating Heat Flux and Electric Current Simultaneously, Phys. Rev. Lett. 113, 205501 (2014).

[15] M. Moccia, G. Castaldi, S. Savo, Y. Sato, and V. Galdi, Independent Manipulation of Heat and Electrical Current via Bifunctional Metamaterials, Phys. Rev. X 4, 021025 (2014).

[16] A. Alu and N. Engheta, Achieving Transparency with Plasmonic and Metamaterial Coatings, Phys. Rev. E 72, 016623 (2005).

[17] A. Alu, Mantle Cloak: Invisibility Induced by a Surface, Phys. Rev. B 80, 245115 (2009).

[18] W. Cai, U. K. Chettiar, A. V. Kildishev, V. M. Shalaev, and G. W. Milton, Nonmagnetic Cloak with Minimized Scattering, Appl. Phys. Lett. 91, 111105 (2007).

[19] M. Yan, Z. Ruan, and M. Qiu, Scattering Characteristics of Simplified Cylindrical Invisibility Cloaks, Opt. Express 15, 17772 (2007).

[20] N. Landy and D. R. Smith, A Full-Parameter Unidirectional Metamaterial Cloak for Microwaves, Nat. Mater. 12, 25 (2013).

[21] L. Xu and H. Chen, Conformal Transformation Optics, Nat. Photonics 9, 15 (2015).

[22] J. Li and J. B. Pendry, Hiding under the Carpet: A New Strategy for Cloaking, Phys. Rev. Lett. 101, 203901 (2008).

[23] R. Liu, C. Ji, J. J. Mock, J. Y. Chin, T. J. Cui, and D. R. Smith, Broadband Ground-Plane Cloak, Science 323, 366 (2009).

[24] M. Selvanayagam and G. V. Eleftheriades, Experimental Demonstration of Active Electromagnetic Cloaking, Phys. Rev. X 3, 041011 (2013).
[25] W. J. M. Kort-Kamp, F. S. S. Rosa, F. A. Pinheiro, and C. Farina, Tuning Plasmonic Cloaks with an External Magnetic Field, Phys. Rev. Lett. 111, 215504 (2013).

[26] Y. Li, X. Shen, Z. Wu, J. Huang, Y. Chen, Y. Ni, and J. Huang, Temperature-Dependent Transformation Thermotics: From Switchable Thermal Cloaks to Macroscopic Thermal Diodes, Phys. Rev. Lett. 115, 195503 (2015).

[27] W. X. Jiang, C. Y. Luo, S. Ge, C. Qiu, and T. J. Cui, An Optically Controllable Transformation-dc Illusion Device, Adv. Mater. 27, 4628 (2015).

[28] Q. Zhao, J. Zhou, F. Zhang, and D. Lippens, Mie ResonanceBased Dielectric Metamaterials, Mater. Today 12, 60 (2009).

[29] Q. Zhao, L. Kang, B. Du, H. Zhao, Q. Xie, X. Huang, B. Li, J. Zhou, and L. Li, Experimental Demonstration of Isotropic Negative Permeability in a Three-Dimensional Dielectric Composite, Phys. Rev. Lett. 101, 027402 (2008).

[30] Q. Zhao, Z. Q. Xiao, F. L. Zhang, J. M. Ma, M. Qiao, Y. G. Meng, C. W. Lan, B. Li, J. Zhou, P. Zhang, N. H. Shen, T. Koschny, and C.M. Soukoulis, Tailorable Zero-Phase Delay of Subwavelength Particles Toward Miniaturized Wave Manipulation Devices, Adv. Mater. 27, 6187 (2015).

[31] See Supplemental Material at http://link.aps.org/ supplemental/10.1103/PhysRevX.7.011033 for the details on the coordinate transformation theory for the one-directional cloak, the design of the dielectric cuboids, the extension of the unidirectional cloak, the demonstration of electromagnetic parameters, and the fabricated sample and experimental measurement.

[32] J. C. Sethares and S. J. Naumann, Design of Microwave Dielectric Resonators, IEEE Trans. Microwave Theory Tech. 14, 2 (1966).

[33] J. B. Pendry, A. J. Holden, D. J. Robbins, and W. J. Stewart, Magnetism from Conductors and Enhanced Nonlinear Phenomena, IEEE Trans. Microwave Theory Tech. 47, 2075 (1999).

[34] A. Sihvola, Metamaterials and Depolarization Factors, Prog. Electromagn. Res. 51, 65 (2005).

[35] Q. Zhao, B. Du, L. Kang, H. Zhao, Q. Xie, B. Li, X. Zhang, J. Zhou, L. Li, and Y.G. Meng, Tunable Negative Permeability in an Isotropic Dielectric Composite, Appl. Phys. Lett. 92, 051106 (2008).

[36] A. J. Bosman and E. E. Havinga, Temperature Dependence of Dielectric Constants of Cubic Ionic Compounds, Phys. Rev. 129, 1593 (1963).

[37] G. Velu, G. Houzet, L. Burgnies, J. C. Carru, A. Marteau, K. Blary, D. Lippens, P. Mounaix, M. Tondusson, and E. Nguema, Electrical Characterizations of Paraelectric BST Thin Films up to $1 \mathrm{THz}$ : Realization of Microwave Phase Shifters, Ferroelectrics 353, 29 (2007).

[38] D. P. Gaillot, C. Croenne, and D. Lippens, An All-Dielectric Route for Terahertz Cloaking, Opt. Express 16, 3986 (2008).

[39] P. Kužel, F. Kadlec, H. Němec, R. Ott, E. Hollmann, and N. Klein, Dielectric Tunability of $\mathrm{SrTiO}_{3}$ Thin Films in the Terahertz Range, Appl. Phys. Lett. 88, 102901 (2006).

[40] J. H. Haeni et al., Room-Temperature Ferroelectricity in Strained $\mathrm{SrTiO}_{3}$, Nature (London) 430, 758 (2004). 